\title{
NON-SOLVABLE GRAPHS OF GROUPS
}

\author{
P. BHOWAL, R. K. NATH AND D. NONGSIANG
}

\begin{abstract}
Let $G$ be a group and $\operatorname{Sol}(G)=\{x \in G:\langle x, y\rangle$ is solvable for all $y \in$ $G\}$. We associate a graph $\mathcal{N S}_{G}$ (called the non-solvable graph of $G$ ) with $G$ whose vertex set is $G \backslash \operatorname{Sol}(G)$ and two distinct vertices are adjacent if they generate a nonsolvable subgroup. In this paper we study many properties of $\mathcal{N} \mathcal{S}_{G}$. In particular, we obtain results on vertex degree, cardinality of vertex degree set, graph realization, domination number, vertex connectivity, independence number and clique number of $\mathcal{N S}_{G}$. We also consider two groups $G$ and $H$ having isomorphic non-solvable graphs and derive some properties of $G$ and $H$. Finally, we conclude this paper by showing that $\mathcal{N S}_{G}$ is neither planar, toroidal, double-toroidal, triple-toroidal nor projective.
\end{abstract}

\section{INTRODUCTION}

Let $G$ be a group (finite/infinite) and $\operatorname{Sol}(G)=\{x \in G:\langle x, y\rangle$ is solvable for all $y \in$ $G\}$. Note that $\operatorname{Sol}(G)=\underset{x \in G}{\bigcap} \operatorname{Sol}_{G}(x)$ where $\operatorname{Sol}_{G}(x)=\{g \in G:\langle g, x\rangle$ is solvable $\}$ is called solvabilizer of $x$ in $G$. In general, $\operatorname{Sol}_{G}(x)$ is not a subgroup of $G$. However, it was shown, in [9], that $\operatorname{Sol}(G)$ is the solvable radical of $G$ if $G$ is a finite group. That is, if $G$ is a finite group then $\operatorname{Sol}(G)$ is the unique largest solvable normal subgroup of $G$. We associate a simple graph $\mathcal{N S}_{G}$ with $G$, called the non-solvable graph of $G$ whose vertex set is $G \backslash \operatorname{Sol}(G)$ and two distinct vertices $x$ and $y$ are adjacent if and only if $\langle x, y\rangle$ is not solvable. The non-solvable graph of $G$ was introduced in [12] and studied in [12, 4]. The complement of $\mathcal{N S}_{G}$, known as solvable graph of $G$, is considered in [5] recently. The concept of non-solvable graph of a group is an extension of non-nilpotent graph and hence non-commuting graph of groups. The non-nilpotent and non-commuting graph of finite groups are studied extensively in [2, 14] and [1, 3, 7, 8, 15] respectively.

We write $V(\Gamma)$ to denote the vertex set of a graph $\Gamma$. The degree of a vertex $x \in V(\Gamma)$ denoted by $\operatorname{deg}(x)$ is defined to be the number of vertices adjacent to $x$ and $\operatorname{deg}(\Gamma)=\{\operatorname{deg}(x): x \in V(\Gamma)\}$ is the vertex degree set of $\Gamma$. In Section 2, we shall study some properties of degree of a vertex and vertex degree set of $\mathcal{N} \mathcal{S}_{G}$. In this section, we also obtain some bounds for solvability degree of a finite group and one of which is better than an existing bound, in particular [5, Theorem 4.3]. The

2010 Mathematics Subject Classification. Primary 20D60; Secondary 05C25.

Key words and phrases. non-Solvable graph; Finite group. 
solvability degree of a finite group $G$ is the probability that a randomly chosen pair of elements of $G$ generate a solvable group (see [11, 5, 17]).

It is known that $\mathcal{N} \mathcal{S}_{G}$ is neither a tree nor a complete graph (see [12, 4]). In Section 3, we shall show that $\mathcal{N S}_{G}$ is not bipartite, more generally it is not complete multi-partite. We shall also show that $\mathcal{N S}_{G}$ is hamiltonian for some classes of finite groups. In Sections 4-6, we shall obtain several results regarding domination number, vertex connectivity, independence number and clique number of $\mathcal{N} \mathcal{S}_{G}$. In section 7 , we shall consider two groups $G$ and $H$ having isomorphic non-solvable graphs and derive some properties of $G$ and $H$. It was shown in [12] that $\mathcal{N} \mathcal{S}_{G}$ is not planar. In the last section, we shall show that the genus of $\mathcal{N} \mathcal{S}_{G}$ is greater or equal to 4 . Hence, $\mathcal{N S}_{G}$ is neither planar, toroidal, double-toroidal nor triple-toroidal. We conclude this paper by showing that $\mathcal{N} \mathcal{S}_{G}$ is not projective.

Let $U$ be a nonempty subset of the vertex set of a graph $\Gamma$. The induced subgraph of $\Gamma$ on $U$ is defined to be the graph $\Gamma[U]$ in which the vertex set is $U$ and the edge set consists precisely those edges in $\Gamma$ whose endpoints lie in $U$. For any non-empty subset $S$ of $V(\Gamma)$, we also write $\Gamma \backslash S$ to denote $\Gamma[V(\Gamma) \backslash S]$.

\section{VERTEX DEGREE AND CARDinAlity OF VERTEX DEGREE SET}

The neighborhood of a vertex $x$ in a graph $\Gamma$, denoted by $\operatorname{nbd}(x)$, is defined to be the set of all vertices adjacent to $x$ and so $\operatorname{deg}(x)=|\operatorname{nbd}(x)|$. It is easy to see that $\operatorname{deg}(x)=|G|-\left|\operatorname{Sol}_{G}(x)\right|$ for any vertex $x$ in the non-solvable graph $\mathcal{N S}_{G}$ of the group $G$. In [12, Hai-Reuven have shown that

$$
6 \leq \operatorname{deg}(x) \leq|G|-|\operatorname{Sol}(G)|-2
$$

for any $x \in G \backslash \operatorname{Sol}(G)$. In this section, we first obtain some bounds for $P_{s}(G)$ using (11). Recall that $P_{s}(G)$ is the solvability degree of a finite group $G$ defined by the ratio

$$
P_{s}(G)=\frac{\mid\{(u, v) \in G \times G:\langle u, v\rangle \text { is solvable }\} \mid}{|G|^{2}} .
$$

It is worth mentioning that several properties of $P_{s}(G)$ including some bounds are studied in [11, 5, 17]. The following result gives a connection between $P_{s}(G)$ and the number of edges in $\mathcal{N} \mathcal{S}_{G}$.

Lemma 2.1. If $G$ is a finite non-solvable group then

$$
\sum_{x \in G \backslash \operatorname{Sol}(G)} \operatorname{deg}(x)=|G|^{2}\left(1-P_{S}(G)\right) .
$$

Proof. Let $U=\{(x, y) \in G \times G:\langle x, y\rangle$ is not solvable $\}$. Then

$$
|U|=|G \times G|-\mid\left.\{(x, y) \in G \times G:\langle x, y\rangle \text { is solvable }\}|=| G\right|^{2}-P_{S}(G)|G|^{2} .
$$

Note that

$$
|U|=2 \times \text { number of edges in } \mathcal{N S}_{G}=\sum_{x \in G \backslash \operatorname{Sol}(G)} \operatorname{deg}(x) \text {. }
$$


Hence the result follows.

Now we obtain the following bounds for $P_{S}(G)$.

Theorem 2.2. If $G$ is a finite non-solvable group then

$$
\frac{2(|G|-|\operatorname{Sol}(G)|)}{|G|^{2}}+\frac{2|\operatorname{Sol}(G)|}{|G|}-\frac{|\operatorname{Sol}(G)|^{2}}{|G|^{2}} \leq P_{S}(G) \leq 1-\frac{6(|G|-|\operatorname{Sol}(G)|)}{|G|^{2}} .
$$

Proof. By Lemma 2.1 and (11), we have

$$
6(|G|-|\operatorname{Sol}(G)|) \leq|G|^{2}\left(1-P_{S}(G)\right) \leq(|G|-|\operatorname{Sol}(G)|)(|G|-|\operatorname{Sol}(G)|-2)
$$

and hence the result follows on simplification.

It was shown in [5, Theorem 4.3] that

$$
P_{S}(G) \geq \frac{2(|G|-|\operatorname{Sol}(G)|)}{|G|^{2}}+\frac{|\operatorname{Sol}(G)|}{|G|} .
$$

Note that $\frac{|\operatorname{Sol}(G)|}{|G|}-\frac{|\operatorname{Sol}(G)|^{2}}{|G|^{2}}>0$ for any finite non-solvable group $G$. Hence, the lower bound obtained in Theorem 2.2 for $P_{S}(G)$ is better than (2).

It was also shown, in [12], that $\left|\operatorname{deg}\left(\mathcal{N S}_{G}\right)\right| \neq 2$, where $\operatorname{deg}\left(\mathcal{N S}_{G}\right)$ is the vertex degree set of $\mathcal{N S}_{G}$. However, we observe that the cardinality of $\operatorname{deg}\left(\mathcal{N S}_{G}\right)$ may be equal to 3. In this section, we shall obtain a class of groups $G$ such that $\left|\operatorname{deg}\left(\mathcal{N} \mathcal{S}_{G}\right)\right|=$ 3. Note that $\operatorname{deg}\left(\mathcal{N S}_{A_{5}}\right)=\{24,36,50\}$. More generally, we have the following result.

Proposition 2.3. Let $S$ be any finite solvable group. Then $\left|\operatorname{deg}\left(\mathcal{N S}_{A_{5} \times S}\right)\right|=3$.

The proof of Proposition 2.3 follows from the fact that $\left|\operatorname{deg}\left(\mathcal{N S}_{A_{5}}\right)\right|=3$ and the result given below.

Lemma 2.4. Let $G$ be a finite non-solvable group and $S$ be any finite solvable group. Then $\left|\operatorname{deg}\left(\mathcal{N S}_{G}\right)\right|=\left|\operatorname{deg}\left(\mathcal{N S}_{G \times S}\right)\right|$.

Proof. Let $(x, s),(y, t) \in G \times S$, then $\langle(x, s),(y, t)\rangle \subseteq\langle x, y\rangle \times\langle s, t\rangle$. Therefore, $\langle(x, s),(y, t)\rangle$ is solvable if and only if $\langle x, y\rangle$ is solvable. Also, $\operatorname{Sol}_{G \times S}((x, s))=$ $\operatorname{Sol}_{G}(x) \times S$ and hence $\operatorname{nbd}((x, s))=\operatorname{nbd}(x) \times S$. That is, $\operatorname{deg}((x, s))=|S| \operatorname{deg}(x)$. This completes the proof.

Now we state the main result of this section.

Theorem 2.5. If $G$ is a finite non-solvable group such that $G / \operatorname{Sol}(G) \cong A_{5}$ then $\left|\operatorname{deg}\left(\mathcal{N S}_{G}\right)\right|=3$.

To prove this theorem we need the following results.

Lemma 2.6. Let $H$ be a subgroup of a finite group $G$ and $x, y \in G$.

(a) If $H$ is solvable then $\langle H, \operatorname{Sol}(G)\rangle$ is also solvable subgroup of $G$.

(b) If $\langle x, y\rangle$ is solvable then $\langle x u, y v\rangle$ is also solvable for all $u, v \in \operatorname{Sol}(G)$.

(c) If $\langle x, y\rangle$ is not solvable then $\langle x u, y v\rangle$ is not solvable for all $u, v \in \operatorname{Sol}(G)$. 
Proof. Part (a) is proved in [5, Lemma 3.1]. Part (b) follows from part (a). Also, note that parts (b) and (c) are equivalent.

Lemma 2.7. Let $G$ be a finite group and $x, y \in G$. Then $\langle x \operatorname{Sol}(G), y \operatorname{Sol}(G)\rangle$ is solvable if and only if $\langle x, y\rangle$ is solvable.

Proof. Let $H=\langle x, y\rangle$ and $Z=\operatorname{Sol}(G)$. Note that $\langle x Z, y Z\rangle=\frac{H Z}{Z}$. Suppose $\langle x Z, y Z\rangle$ is solvable. Then $\frac{H Z}{Z}$ is solvable. Since $Z \subset \operatorname{Sol}(H Z)$ and $Z$ is a normal subgroup of $H Z$, by [12, Lemma 2.11 (3)], we have

$$
\frac{\operatorname{Sol}_{H Z}(x)}{Z}=\operatorname{Sol}_{\frac{H Z}{Z}}(x Z)=\frac{H Z}{Z} .
$$

Therefore, $\operatorname{Sol}_{H Z}(x)=H Z$. In particular, $\operatorname{Sol}_{H}(x)=H$ and so $H$ is solvable.

If $H$ is solvable then, by Lemma 2.6 (b), $\operatorname{Sol}_{H Z}(x)=H Z$ for all $x \in H Z$. Thus $H Z$ is solvable and so $\frac{H Z}{Z}$ is solvable. Hence, $\left\langle x_{i} Z, x_{j} Z\right\rangle$ is solvable for $x_{i}, x_{j} \in H Z$ and so $\langle x Z, y Z\rangle$ is solvable.

Proposition 2.8. Let $G$ be a finite non-solvable group. Then for all $x \in G \backslash \operatorname{Sol}(G)$ we have

$$
\operatorname{deg}(x)=\operatorname{deg}(x \operatorname{Sol}(G))|\operatorname{Sol}(G)| \text {. }
$$

Proof. Let $y \in \operatorname{nbd}(x)$. By Lemma 2.6, we have $y z \in \operatorname{nbd}(x)$ for all $z \in \operatorname{Sol}(G)$. Thus $\operatorname{nbd}(x)$ is a union of distinct cosets of $\operatorname{Sol}(G)$. Let $\operatorname{nbd}(x)=y_{1} \operatorname{Sol}(G) \cup$ $y_{2} \operatorname{Sol}(G) \cup \cdots \cup y_{n} \operatorname{Sol}(G)$. Then $\operatorname{deg}(x)=n|\operatorname{Sol}(G)|$. By Lemma 2.7, we have $\left\langle x \operatorname{Sol}(G), y_{i} \operatorname{Sol}(G)\right\rangle$ is not solvable if and only if $\left\langle x, y_{i}\right\rangle$ is not solvable. Therefore, $\operatorname{nbd}(x \operatorname{Sol}(G))=\left\{y_{1} \operatorname{Sol}(G), y_{2} \operatorname{Sol}(G), \ldots, y_{n} \operatorname{Sol}(G)\right\}$ in $\mathcal{N S}_{G / \operatorname{Sol}(G)}$. Hence, $\operatorname{deg}(x \operatorname{Sol}(G))=n$ and the result follows.

As a consequence of Proposition 2.8 we have the following corollary.

Corollary 2.9. Let $G$ be a finite non-solvable group. Then $\left|\operatorname{deg}\left(\mathcal{N S}_{G / \operatorname{Sol}(G)}\right)\right|=$ $\left|\operatorname{deg}\left(\mathcal{N S}_{G}\right)\right|$.

Proof of Theorem 2.5; Note that $G / \operatorname{Sol}(G) \cong A_{5}$ implies $\mathcal{N} \mathcal{S}_{G / \operatorname{Sol}(G)} \cong \mathcal{N} \mathcal{S}_{A_{5}}$. Therefore

$$
\left|\operatorname{deg}\left(\mathcal{N S}_{G / \operatorname{Sol}(G)}\right)\right|=\left|\operatorname{deg}\left(\mathcal{N S}_{A_{5}}\right)\right|=3
$$

Hence, the result follows from Corollary 2.9.

We conclude this section with the following upper bound for $\left|\operatorname{deg}\left(\mathcal{N} \mathcal{S}_{G}\right)\right|$.

Theorem 2.10. If $G$ is a finite non-solvable group having $n$ distinct solvabilizers then $\left|\operatorname{deg}\left(\mathcal{N S}_{G}\right)\right| \leq n-1$.

Proof. Let $G, X_{1}, X_{2}, \ldots, X_{n-1}$ be the distinct solvabilizers of $G$ where $\operatorname{Sol}_{G}\left(x_{i}\right)=X_{i}$ for some $x_{i} \in G \backslash \operatorname{Sol}(G)$ and $i=1,2, \ldots, n-1$. Then

$$
\operatorname{deg}\left(\mathcal{N S}_{G}\right)=\left\{|G|-\left|X_{1}\right|,|G|-\left|X_{2}\right|, \ldots,|G|-\left|X_{n-1}\right|\right\} .
$$

Hence, the result follows. 


\section{Graph Realization}

In [12, it was shown that $\mathcal{N S}_{G}$ is connected with diameter two. It is also shown that $\mathcal{N S}_{G}$ is not regular and hence not a complete graph. Recently, Akbari [4 have shown that $\mathcal{N} \mathcal{S}_{G}$ is not a tree. In this section, we shall show that $\mathcal{N} \mathcal{S}_{G}$ is not a complete multi-partite graph. We shall also show that $\mathcal{N} \mathcal{S}_{G}$ is hamiltonian for some groups. The following results are useful in this regard.

Lemma 3.1. [12] Let $G$ be a finite group. Then $G$ is solvable if and only if $\operatorname{Sol}_{G}(x)$ is a subgroup of $G$ for all $x \in G$.

Theorem 3.2. [9] Let $G$ be a finite non-solvable group and $x, y \in G \backslash \operatorname{Sol}(G)$. Then there exists $s \in G \backslash \operatorname{Sol}(G)$ such that $\langle x, s\rangle$ and $\langle y, s\rangle$ are not solvable.

Theorem 3.3. Let $G$ be a finite non-solvable group. Then $\mathcal{N S}_{G}$ is not a complete multi-partite graph. In particular, $\mathcal{N S}_{G}$ is not a complete bipartite graph.

Proof. Suppose $\mathcal{N S}_{G}$ is a complete multi-partite graph. Let $X_{1}, X_{2}, \ldots, X_{n}$ be the partite sets. Let $x \in G \backslash \operatorname{Sol}(G)$, then $x \in X_{i}$ for some $i$ and $\operatorname{Sol}_{G}(x)=\operatorname{Sol}(G) \cup X_{i}$. Let $y, z \in \operatorname{Sol}_{G}(x)$. Then $\langle y, z\rangle$ is solvable and $y z \in \operatorname{Sol}_{G}(y)=\operatorname{Sol}_{G}(x)$. Thus $\operatorname{Sol}_{G}(x)$ is a subgroup of $G$. By Lemma 3.1, $G$ is solvable, a contradiction. Hence, the result follows.

Theorem 3.4. Let $G$ be a finite non-solvable group. Then $\mathcal{N S}_{G}$ is not a bipartite graph.

Proof. Suppose $\mathcal{N S}_{G}$ is a bipartite graph. Let $X, Y$ be the partite sets. Let $x \in X$ and $y \in Y$. Then, by Theorem 3.2 , there exists $z \in G \backslash \operatorname{Sol}(G)$ such that $\langle x, z\rangle$ and $\langle y, z\rangle$ are not solvable. Therefore, $z \notin X \cup Y$, a contradiction. Hence the result follows.

Theorem 3.5. Let $G$ be a finite non-solvable group such that $\left|\operatorname{Sol}_{G}(x)\right| \leq \frac{|G|}{2}$ for all $x \in G \backslash \operatorname{Sol}(G)$. Then $\mathcal{N S}_{G}$ is hamiltonian.

Proof. Note that $\operatorname{deg}(x)=|G|-\left|\operatorname{Sol}_{G}(x)\right|$ for all $x \in G \backslash \operatorname{Sol}(G)$. Since $\left|\operatorname{Sol}_{G}(x)\right| \leq \frac{|G|}{2}$ for all $x \in G \backslash \operatorname{Sol}(G)$ we have $|G| \geq 2\left|\operatorname{Sol}_{G}(x)\right|$. Thus, it follows that $\operatorname{deg}(x)>$ $(|G|-|\operatorname{Sol}(G)|) / 2$. Therefore by Dirac's Theorem [6, p. 54], $\mathcal{N} \mathcal{S}_{G}$ is hamiltonian.

Corollary 3.6. The non-solvable graph of the group $P S L(3,2) \rtimes \mathbb{Z}_{2}, A_{6}$ and $P S L(2,8)$ are hamiltonian.

Proof. The result follows from Theorem 3.5 using the fact that $\left|\operatorname{Sol}_{G}(x)\right| \leq \frac{|G|}{2}$ for all $x \in G \backslash \operatorname{Sol}(G)$ where $G=P S L(3,2) \rtimes \mathbb{Z}_{2}, A_{6}$ and $P S L(2,8)$.

The following result shows that there is a group $G$ with $\left|\operatorname{Sol}_{G}(x)\right|>|G| / 2$ for some $x \in G \backslash \operatorname{Sol}(G)$ such that $\mathcal{N} \mathcal{S}_{G}$ is hamiltonian.

Proposition 3.7. The non-solvable graph of $A_{5}$ is hamiltonian. 
Proof. For any two vertex $a$ and $b$ we write $a \sim b$ if $a$ is adjacent to $b$. It can be verified that

$(1,5,4,3,2) \sim(1,3)(2,5) \sim(2,3,4) \sim(1,4)(3,5) \sim(2,5,4) \sim(1,2)(3,4) \sim$ $(1,5,4) \sim(2,5)(3,4) \sim(1,3,5) \sim(1,4)(2,5) \sim(2,4,3) \sim(1,3)(4,5) \sim(1,2,5) \sim$ $(1,4)(2,3) \sim(3,5,4) \sim(1,5)(2,4) \sim(1,2,3) \sim(1,5)(3,4) \sim(2,3,5) \sim(1,4,2) \sim$ $(2,3)(4,5) \sim(1,5,2) \sim(2,4)(3,5) \sim(1,4,5) \sim(1,2)(3,5) \sim(1,3,4) \sim(1,2)(4,5) \sim$ $(1,5,3) \sim(1,4,2,5,3) \sim(1,3,2) \sim(3,4,5) \sim(1,3)(2,4) \sim(2,5,3) \sim(1,2,4) \sim$ $(1,5)(2,3) \sim(2,4,5) \sim(1,4,3) \sim(1,3,5,2,4) \sim(1,4,5,3,2) \sim(1,2,3,4,5) \sim$ $(1,2,4,3,5) \sim(1,5,3,2,4) \sim(1,4,5,2,3) \sim(1,5,4,2,3) \sim(1,3,4,5,2) \sim(1,5,3,4,2)$ $\sim(1,3,2,4,5) \sim(1,3,2,5,4) \sim(1,2,4,5,3) \sim(1,2,5,4,3) \sim(1,5,2,3,4) \sim$ $(1,2,3,5,4) \sim(1,4,3,2,5) \sim(1,4,3,5,2) \sim(1,3,4,2,5) \sim(1,4,2,3,5) \sim(1,5,2,4,3)$ $\sim(1,3,5,4,2) \sim(1,2,5,3,4) \sim(1,5,4,3,2)$

is a hamiltonian cycle of $\mathcal{N S}_{A_{5}}$. Hence, $\mathcal{N S}_{A_{5}}$ is hamiltonian.

We conclude this section with the following question.

Question 3.8. Is $\mathcal{N S}_{G}$ hamiltonian for any finite non-solvable group G?

\section{Domination number And Vertex COnNeCtivity}

For a graph $\Gamma$ and a subset $S$ of the vertex set $V(\Gamma)$ we write $N_{\Gamma}[S]=S \cup$ $\left(\cup_{x \in S} \operatorname{nbd}(x)\right)$. If $N_{\Gamma}[S]=V(\Gamma)$ then $S$ is said to be a dominating set of $\Gamma$. The domination number of $\Gamma$, denoted by $\lambda(\Gamma)$, is the minimum cardinality of dominating sets of $\Gamma$. In this section, we shall obtain a few results regarding $\lambda\left(\mathcal{N S}_{G}\right)$.

Proposition 4.1. Let $G$ be a finite non-solvable group. Then $\lambda\left(\mathcal{N S}_{G}\right) \neq 1$.

Proof. Let $\{x\}$ be a dominating set for $\mathcal{N S}_{G}$. If $\operatorname{Sol}(G)$ contains a non-trivial element $z$ then $x z$ is adjacent to $x$, a contradiction. Hence, $|\operatorname{Sol}(G)|=1$.

If $o(x) \neq 2$ then $x$ is adjacent to $x^{-1}$, which is a contradiction. Hence, $o(x)=2$ and so $x \in P_{2}$, for some Sylow 2-subgroup $P_{2}$ of $G$. Since $|\operatorname{Sol}(G)|=1$ and $x$ is adjacent to all vertices of $\mathcal{N S}_{G}$ we have $\operatorname{Sol}_{G}(x)=\langle x\rangle$. Also, $P_{2} \subseteq \operatorname{Sol}_{G}(x)$ and so $P_{2}=\langle x\rangle$. If $Q_{2}$ is another Sylow 2-subgroup of $G$ then $\left|Q_{2}\right|=2$ and so $\left\langle P_{2}, Q_{2}\right\rangle$ is a dihedral group and hence solvable. That is, $x$ is not adjacent to $y \in Q_{2}, y \neq 1$, which is a contradiction. Thus it follows that $P_{2}$ is normal in $G$. Let $g \in G \backslash P_{2}$. Then $g x g^{-1}=x$, that is $x g=g x$ and so $x \in Z(G)$, which is a contradiction. Hence, the result follows.

Using GAP [18] it can be seen that $\lambda\left(A_{5}\right)=\lambda\left(S_{5}\right)=4$. In fact $\{(3,4,5),(1,2,3,4,5)$, $(1,2,4,5,3),(1,5)(2,4)\}$ and $\{(4,5),(1,2)(3,4,5),(1,3)(2,4,5),(1,5)(2,4)\}$ are dominating sets for $A_{5}$ and $S_{5}$ respectively. At this point we would like to ask the following question.

Question 4.2. Is there any finite non-solvable group $G$ such that $\lambda\left(\mathcal{N S}_{G}\right)=2,3$ ?

Proposition 4.3. Let $G$ be a non-solvable group. Then a subset $S$ of $V\left(\mathcal{N S}_{G}\right)$ is a dominating set if and only if $\operatorname{Sol}_{G}(S) \subset \operatorname{Sol}(G) \cup S$. 
Proof. Suppose $S$ is a dominating set. If $a \notin \operatorname{Sol}(G) \cup S$ then, by definition of dominating set, there exists $x \in S$ such that $\langle x, a\rangle$ is not solvable. Thus $a \notin \operatorname{Sol}_{G}(S)$. It follows that $\operatorname{Sol}_{G}(S) \subset S \cup \operatorname{Sol}(G)$.

Now assume that $\operatorname{Sol}_{G}(S) \subset \operatorname{Sol}(G) \cup S$. If $a \notin \operatorname{Sol}(G) \cup S$, then by hypothesis, $a \notin \operatorname{Sol}_{G}(S)$. Therefore, $a$ is adjacent to at least one element of $S$. This completes the proof.

The vertex connectivity of a connected graph $\Gamma$, denoted by $\kappa(\Gamma)$, is defined as the smallest number of vertices whose removal makes the graph disconnected. A subset $S$ of the vertices of a connected graph $\Gamma$ is called a vertex cut set, if $\Gamma \backslash S$ is not connected but $\Gamma \backslash H$ is connected for any proper subset $H$ of $S$. We conclude this section with the following result on vertex cut set and vertex connectivity of $\mathcal{N} \mathcal{S}_{G}$.

Proposition 4.4. Let $G$ be a finite non-solvable group and let $S$ be a vertex cut set of $\mathcal{N S}_{G}$. Then $S$ is a union of cosets of $\operatorname{Sol}(G)$. In particular $\kappa\left(\mathcal{N} \mathcal{S}_{G}\right)=t|\operatorname{Sol}(G)|$, where $t>1$ is an integer.

Proof. Let $a \in S$. Then there exist two distinct components $G_{1}, G_{2}$ of $\mathcal{N S}_{G} \backslash S$ and two vertices $x \in V\left(G_{1}\right), y \in V\left(G_{2}\right)$ such that $a$ is adjacent to both $x$ and $y$. By Lemma 2.6, $x$ and $y$ are also adjacent to $a z$ for any $z \in \operatorname{Sol}(G)$, and so $a \operatorname{Sol}(G) \subset S$. Thus $S$ is a union of cosets of $\operatorname{Sol}(G)$. Hence, $\kappa\left(\mathcal{N} \mathcal{S}_{G}\right)=t|\operatorname{Sol}(G)|$, where $t \geq 1$ is an integer.

Suppose that $|S|=\kappa\left(\mathcal{N} \mathcal{S}_{G}\right)$. It follows from the first part that $\kappa\left(\mathcal{N} \mathcal{S}_{G}\right)=t|\operatorname{Sol}(G)|$ for some integer $t \geq 1$. If $t=1$ then $S=b \operatorname{Sol}(G)$ for some element $b \in G \backslash \operatorname{Sol}(G)$. Therefore, there exist two distinct components $G_{1}, G_{2}$ of $\mathcal{N S}_{G} \backslash S$ and $r \in V\left(G_{1}\right)$, $s \in V\left(G_{2}\right)$ such that $b$ is adjacent to both $r$ and $s$. In other words, $\langle b, r\rangle$ and $\langle b, s\rangle$ are not solvable. Suppose that $o(b) \neq 2$. Then the number of integers less than $o(b)$ and relatively prime to it is greater or equal to 2 . Let $1 \neq i \in \mathbb{N} \operatorname{such}$ that $\operatorname{gcd}(i, o(b))=1$. Then

$$
\left\langle b^{i}, r\right\rangle=\langle b, r\rangle \text { and }\left\langle b^{i}, s\right\rangle=\langle b, s\rangle .
$$

Therefore, $b^{i}$ is adjacent to both $r$ and $s$. This is a contradiction since $b^{i} \notin b \operatorname{Sol}(G)$. Hence, $o(b)=2$.

Suppose $x^{\prime} \in V\left(G_{1}\right)$ and $y^{\prime} \in V\left(G_{2}\right)$ are adjacent to $b z$ for some $z \in \operatorname{Sol}(G)$. Then, by Lemma 2.6 (c), $b$ is adjacent to $x^{\prime}$ and $y^{\prime}$. Again, by Lemma 2.7, $x^{\prime} \operatorname{Sol}(G)$ and $y^{\prime} \operatorname{Sol}(G)$ are adjacent to $b \operatorname{Sol}(G)$ in the graph $\mathcal{N S}_{G / \operatorname{Sol}(G)}$. That is, $g \operatorname{Sol}(G)$ and $b \operatorname{Sol}(G)$ are adjacent for all $g \operatorname{Sol}(G) \in V\left(\mathcal{N S}_{G / \operatorname{Sol}(G)}\right)$. Therefore, $\{b \operatorname{Sol}(G)\}$ is a dominating set of $\mathcal{N} \mathcal{S}_{G / \operatorname{Sol}(G)}$ and so $\lambda\left(\mathcal{N S}_{G / \operatorname{Sol}(G)}\right)=1$. Hence, the result follows in view of Proposition 4.1 .

\section{IndEPENDENCE Number}

A subset $X$ of the vertices of a graph $\Gamma$ is called an independent set if the induced subgraph on $X$ has no edges. The maximum size of an independent set in a graph $\Gamma$ 
is called the independence number of $\Gamma$ and it is denoted by $\alpha(\Gamma)$. In this section we consider the following question.

Question 5.1. Suppose $G$ is a non-solvable group such that $\mathcal{N S}_{G}$ has no infinite independent set. Is it true that $\alpha\left(\mathcal{N S}_{G}\right)$ is finite?

It is worth mentioning that Question 5.1] is similar to [1, Question 2.10] and [13, Question 3.17] where Abdollahi et al. and Nongsiang et al. considered non-commuting and non-nilpotent graphs of finite groups respectively. Note that the group considered in [13] in order to answer [13, Question 3.17] negatively, also gives negative answer to Question 5.1. However, the next theorem gives affirmative answer to Question 5.1 for some classes of groups.

Theorem 5.2. Let $G$ be a non-solvable group such that $\mathcal{N S}_{G}$ has no infinite independent sets. If $\operatorname{Sol}(G)$ is a subgroup and $G$ is an Engel, locally finite, locally solvable, a linear group or a 2-group then $G$ is a finite group. In particular $\alpha\left(\mathcal{N S}_{G}\right)$ is finite.

Note that Theorem 5.2 and its proof are similar to [1, Theorem 2.11] and [13, Theorem 3.18].

Proposition 5.3. Let $G$ be a group. Then for every maximal independent set $S$ of $\mathcal{N S}_{G}$ we have

$$
\bigcap_{x \in S} \operatorname{Sol}_{G}(x)=S \cup \operatorname{Sol}(G)
$$

Proof. The result follows from the fact that $S$ is maximal and $S \cup \operatorname{Sol}(G) \subset \operatorname{Sol}_{G}(x)$ for all $x \in S$.

Remark 5.4. Let $R=\{(3,4,5),(1,4)(3,5),(2,5,3)\} \subset A_{5}$. Then $R$ is an independent set of $\mathcal{N S}_{A_{5}}$ and $\langle R\rangle \cong A_{5}$. This shows that a subgroup generated by an independent set may not be solvable. Also there exist a maximal independent set $S$, such that $R \subseteq S$. Since the edge set of $\mathcal{N S}_{A_{5}}$ is non-empty, we have $S \neq A_{5} \backslash \operatorname{Sol}\left(A_{5}\right)$, showing that $S \cup \operatorname{Sol}\left(A_{5}\right)$ is not a subgroup of $A_{5}$. Thus for a finite non-solvable group $G$ and a maximal independent set $S$ of $\mathcal{N S}_{G}, S \cup \operatorname{Sol}(G)$ need not be a subgroup of $G$.

We conclude this section with the following result.

Theorem 5.5. The order of a finite non-solvable group $G$ is bounded by a function of the independence number of its non-solvable graph. Consequently, given a nonnegative integer $k$, there are at the most finitely many finite non-solvable groups whose non-solvable graphs have independence number $k$.

Proof. Let $x \in G$ such that $x, x^{2} \notin \operatorname{Sol}(G)$. Then $x \operatorname{Sol}(G) \cup x^{2} \operatorname{Sol}(G)$ is an independent set of $\mathcal{N S}_{G}$. Thus $|\operatorname{Sol}(G)| \leq \frac{k}{2}$. Let $P$ be a Sylow subgroup of $G$, then $P$ is solvable. Thus it follows that $P \backslash \operatorname{Sol}(G)$ is an independent set of $G$. Hence $|P \backslash \operatorname{Sol}(G)| \leq k$, that is $|P| \leq \frac{3 k}{2}$. Since, the number of primes less or equal to $\frac{3 k}{2}$ is at most $\frac{3 k}{4}$, we have $|G| \leq\left(\frac{3 k}{2}\right)^{\frac{3 k}{4}}$. This completes the proof. 


\section{Clique number of NON-SOlvable GraphS}

A subset of the vertex set of a graph $\Gamma$ is called a clique of $\Gamma$ if it consists entirely of pairwise adjacent vertices. The least upper bound of the sizes of all the cliques of $\Gamma$ is called the clique number of $\Gamma$, and is denoted by $\omega(\Gamma)$. Note that $\omega(\tilde{\Gamma}) \leq \omega(\Gamma)$ for any subgraph $\tilde{\Gamma}$ of $\Gamma$.

Proposition 6.1. Let $G$ be a finite non-solvable group.

(a) If $H$ is a non-solvable subgroup of $G$ then $\omega\left(\mathcal{N S}_{H}\right) \leq \omega\left(\mathcal{N} \mathcal{S}_{G}\right)$.

(b) If $\frac{G}{N}$ is non-solvable then $\omega\left(\mathcal{N S}_{\frac{G}{N}}\right) \leq \omega\left(\mathcal{N S}_{G}\right)$. The equality holds when $N=$ $\operatorname{Sol}(G)$.

Proof. Part (a) follows from the fact that $\mathcal{N} \mathcal{S}_{H}$ is a subgraph of $\mathcal{N S}_{G}$. For part (b), we shall show that $\mathcal{N} \mathcal{S}_{\frac{G}{N}}$ is isomorphic to a subgraph of $\mathcal{N} \mathcal{S}_{G}$.

Let $V\left(\mathcal{N S}_{\frac{G}{N}}\right)=\left\{x_{1} N, x_{2} N, \ldots, x_{n} N\right\}$ and $K=\left\{x_{1}, x_{2}, \ldots, x_{n}\right\}$. Then, for $x_{i} N \in$ $V\left(\mathcal{N} \mathcal{S}_{\frac{G}{N}}\right)$, there exist $x_{j} N \in V\left(\mathcal{N S}_{\frac{G}{N}}\right)$ such that $\left\langle x_{i} N, x_{j} N\right\rangle$ is not solvable. Let $H=\left\langle x_{i}, x_{j}\right\rangle$. Then

$$
\left\langle x_{i} N, x_{j} N\right\rangle=\frac{H N}{N} .
$$

Suppose $H$ is solvable. Then there exists a sub-normal series $\{1\}=H_{0} \leq H_{1} \leq H_{2} \leq$ $\cdots \leq H_{n}=H$, where $H_{i}$ is normal in $H_{i+1}$ and $\frac{H_{i+1}}{H_{i}}$ is abelian for all $i=0,1, \ldots, n-1$. Consider the series $N=H_{0} N \leq H_{1} N \leq \cdots \leq H_{n} N=H N$. We have $H_{i} N$ is normal in $H_{i+1} N$, for if $a n \in H_{i} N$ and $b m \in H_{i+1} N$ then $\operatorname{bman}(b m)^{-1} \in H_{i} N$. Also, $\frac{H_{i+1} N}{H_{i} N}$ is abelian, for if $a, b \in \frac{H_{i+1} N}{H_{i} N}$ then $a=k n_{1}\left(H_{i} N\right)=k\left(H_{i} N\right)$ and $b=$ $\ln _{2}\left(H_{i} N\right)=l\left(H_{i} N\right)$. Therefore, $a b=k l\left(H_{i} N\right)$. Since $H_{i+1} / H_{i}$ is abelian, we have $k H_{i} l H_{i}=l H_{i} k H_{i}$, that is $k l H_{i}=l k H_{i}$. Thus

$$
a b=k l\left(H_{i} N\right)=\left(k l H_{i}\right) N=\left(l k H_{i}\right) N=l k\left(H_{i} N\right)=b a .
$$

Therefore, $\frac{H_{i+1} N}{H_{i} N}$ is abelian. Hence, $H N$ is solvable and so $H N / N=\left\langle x_{i} N, x_{j} N\right\rangle$ is also solvable; which is a contradiction. Therefore, $H$ is non-solvable. Let $L$ be a graph such that $V(L)=K$ and two vertices $x, y$ of $L$ are adjacent if and only if $x N$ and $y N$ are adjacent in $\mathcal{N S}_{\frac{G}{N}}$. Then $L$ is a subgraph of $\mathcal{N S}_{G}[K]$ and hence a subgraph of $\mathcal{N} \mathcal{S}_{G}$. Define a map $\phi: V\left(\mathcal{N S}_{\frac{G}{N}}\right) \rightarrow V(L)$ by $\phi\left(x_{i} N\right)=x_{i}$. Then $\phi$ is one-one and onto. Also two vertices $x_{i} N$ and $x_{j} N$ are adjacent in $\mathcal{N} \mathcal{S}_{\frac{G}{N}}$ if and only if $x_{i}$ and $x_{j}$ are adjacent in $L$. Thus $\mathcal{N S}_{\frac{G}{N}} \cong L$.

If $N=\operatorname{Sol}(G)$ then, by Lemma 2.7, it follows that $\left\{x_{1} \operatorname{Sol}(G), x_{2} \operatorname{Sol}(G), \ldots\right.$, $\left.x_{t} \operatorname{Sol}(G)\right\}$ is a clique of $\mathcal{N} \mathcal{S}_{\frac{G}{\operatorname{Sol}(G)}}$ if and only if $\left\{x_{1}, x_{2}, \ldots, x_{t}\right\}$ is a clique of $\mathcal{N} \mathcal{S}_{G}$. Hence, $\omega\left(\mathcal{N S}_{\frac{G}{\operatorname{Sol}(G)}}\right)=\omega\left(\mathcal{N S}_{G}\right)$.

Theorem 6.2. For any non-solvable group $G$ and a solvable group $S$ we have

$$
\omega\left(\mathcal{N S}_{G}\right)=\omega\left(\mathcal{N S}_{G \times S}\right) .
$$


Proof. Suppose $C$ is a clique of $\mathcal{N S}_{G}$. Let $a, b \in C$, then $\langle a, b\rangle$ is not solvable. Now, $\left\langle\left(a, e_{s}\right),\left(b, e_{s}\right)\right\rangle \cong\langle a, b\rangle$, where $e_{s}$ is the identity element of $S$, and so $\left\langle\left(a, e_{s}\right),\left(b, e_{s}\right)\right\rangle$ is not solvable. Thus $C \times\left\{e_{s}\right\}$ is a clique of $\mathcal{N} \mathcal{S}_{G \times S}$. Now suppose $D$ is a clique of $\mathcal{N S}_{G \times S}$. Let $\left(x, s_{1}\right),\left(y, s_{2}\right) \in D$, where $x \neq y$. Then $\left\langle\left(x, s_{1}\right),\left(y, s_{2}\right)\right\rangle \subseteq\langle x, y\rangle \times\left\langle s_{1}, s_{2}\right\rangle$. Since $S$ is solvable, we have $\left\langle s_{1}, s_{2}\right\rangle$ is solvable. Since $\left\langle\left(x, s_{1}\right),\left(y, s_{2}\right)\right\rangle$ is not solvable, we have $\langle x, y\rangle$ is not solvable. Thus $E=\{x:(x, s) \in D\}$ is a clique of $\mathcal{N} \mathcal{S}_{G}$. Hence, the result follows noting that $|D|=|E|$.

The following lemma is useful in obtaining a lower bound for $\omega\left(\mathcal{N} \mathcal{S}_{G}\right)$.

Lemma 6.3. Let $G$ be a finite non-solvable group. Then there exists an element $x \in G \backslash \operatorname{Sol}(G)$ such that $o(x)$ is a prime greater or equal to 5 .

Proof. Suppose that $1 \neq o(x)=2^{\alpha} 3^{\beta}$ for all $x \in G \backslash \operatorname{Sol}(G)$, where $\alpha$ and $\beta$ are nonzero integers. Then $|G / \operatorname{Sol}(G)|=2^{m} 3^{n}$ for some non-zero integers $m, n$. Therefore, $G / \operatorname{Sol}(G)$ is solvable and so, by Lemma 2.7, $G$ is solvable; a contradiction. This proves the existence of an element $x \in G \backslash \operatorname{Sol}(G)$ such that $o(x)$ is a prime greater or equal to 5 .

Proposition 6.4. Let $G$ be a finite non-solvable group. Then $\omega\left(\mathcal{N} \mathcal{S}_{G}\right) \geq 6$.

Proof. By Lemma 6.3, we have an element $x \in G \backslash \operatorname{Sol}(G)$ such that $o(x)$ is a prime greater or equal to 5 . Let $y \in G \backslash \operatorname{Sol}(G)$ such that $x$ is adjacent to $y$. Then $\left\{x, y, x y, x^{2} y, x^{3} y, x^{4} y\right\}$ is a clique of $\mathcal{N S}_{G}$ and so $\omega\left(\mathcal{N S}_{G}\right) \geq 6$.

The following program in GAP [18] shows that

$$
\omega\left(\mathcal{N S}_{A_{5}}\right)=\omega\left(\mathcal{N S}_{S L(2,5)}\right)=\omega\left(\mathcal{N S}_{\mathbb{Z}_{2} \times A_{5}}\right)=8 \text { and } \omega\left(\mathcal{N S}_{S_{5}}\right)=16 \text {. }
$$

Note that $A_{5}=\operatorname{SmallGroup}(60,5), \quad S L(2,5)=\operatorname{SmallGroup}(120,5), \quad S_{5}=$ $\operatorname{SmallGroup}(120,34)$ and $\mathbb{Z}_{2} \times A_{5}=\operatorname{SmallGroup}(120,35)$. Also $G / \operatorname{Sol}(G) \cong A_{5}$ for $G=A_{5}, S L(2,5)$ and $\mathbb{Z}_{2} \times A_{5}$.

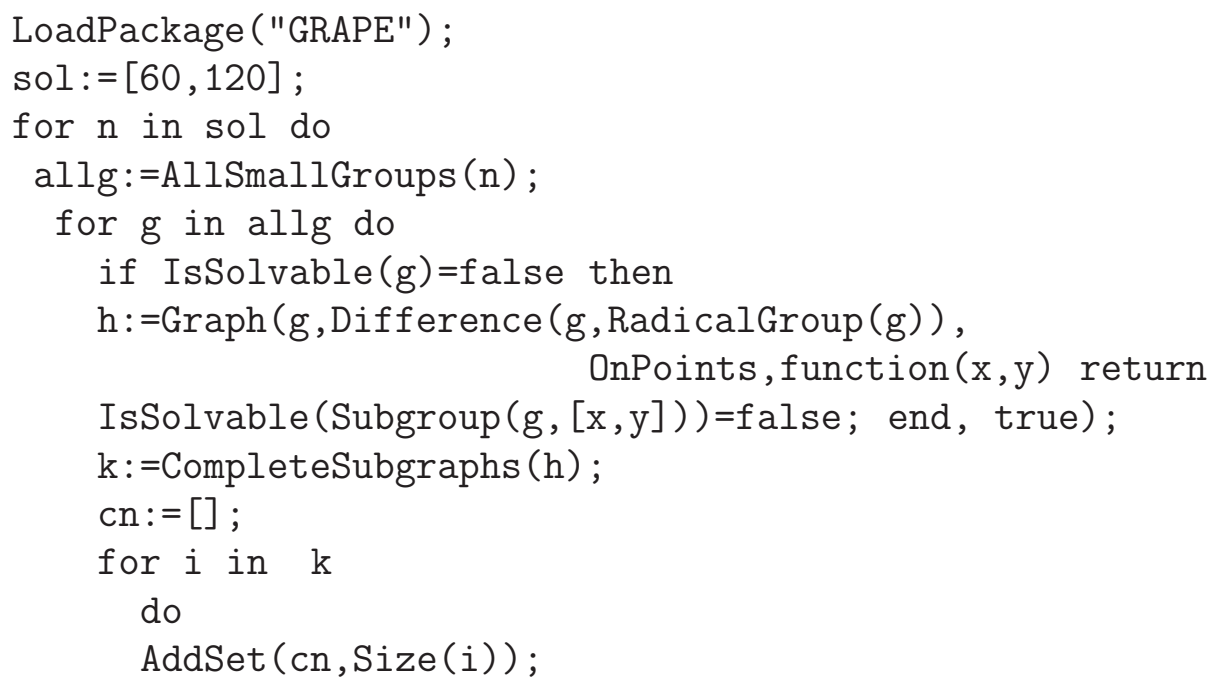




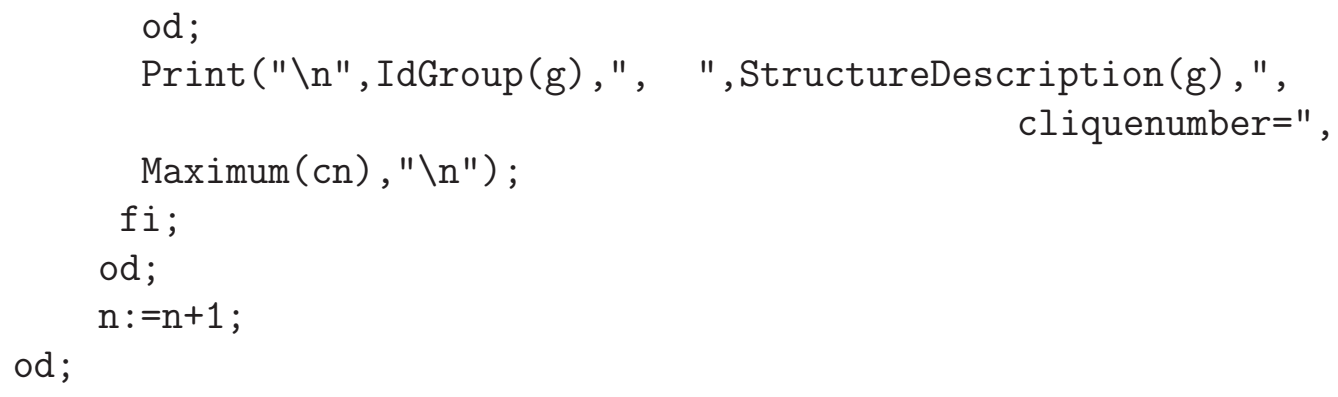

The following program in GAP [18] shows that the clique number of $\mathcal{N} \mathcal{S}_{G}$ for groups of order less or equal to 360 with $G / \operatorname{Sol}(G) \neq A_{5}$ is greater or equal to 9 .

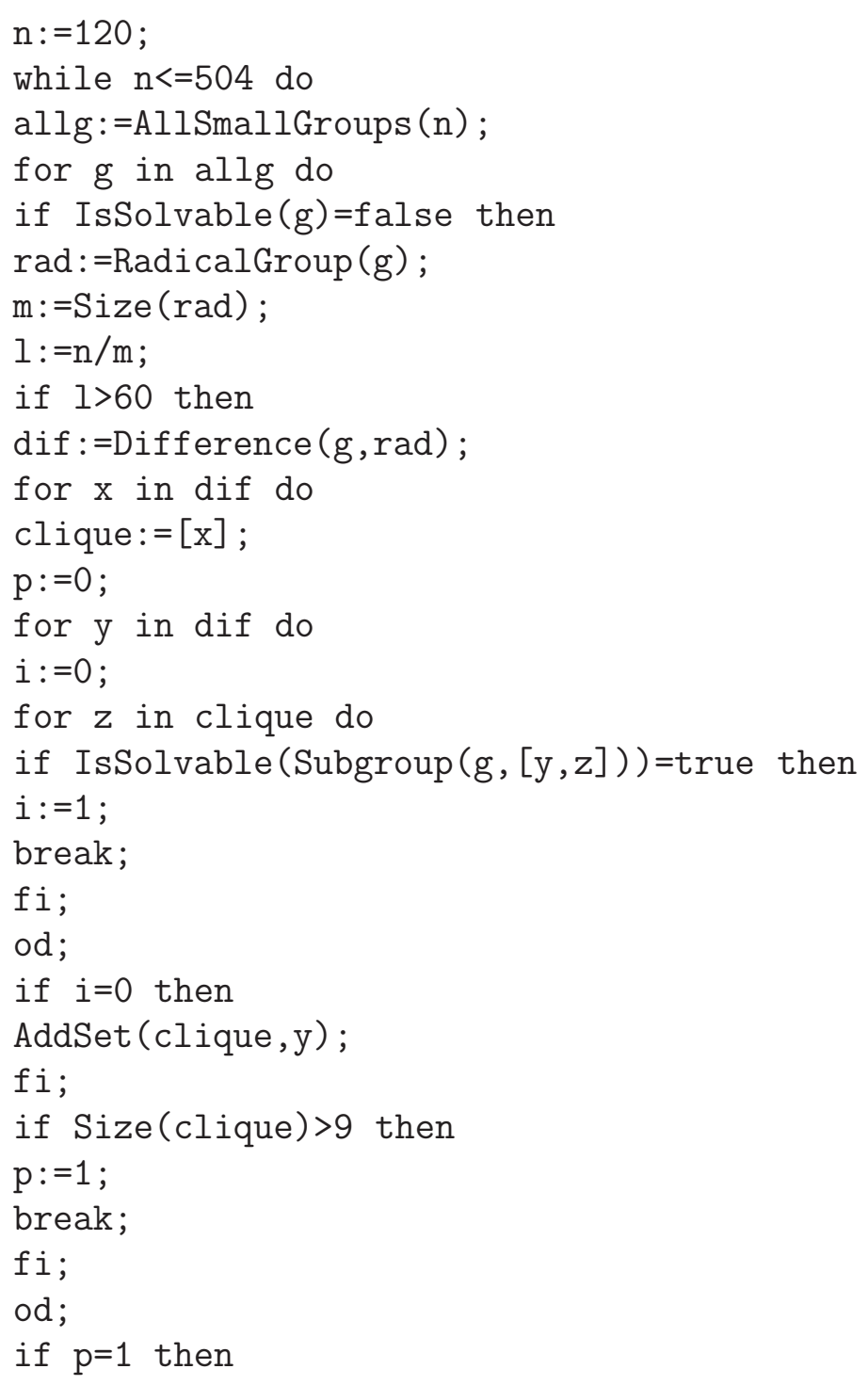




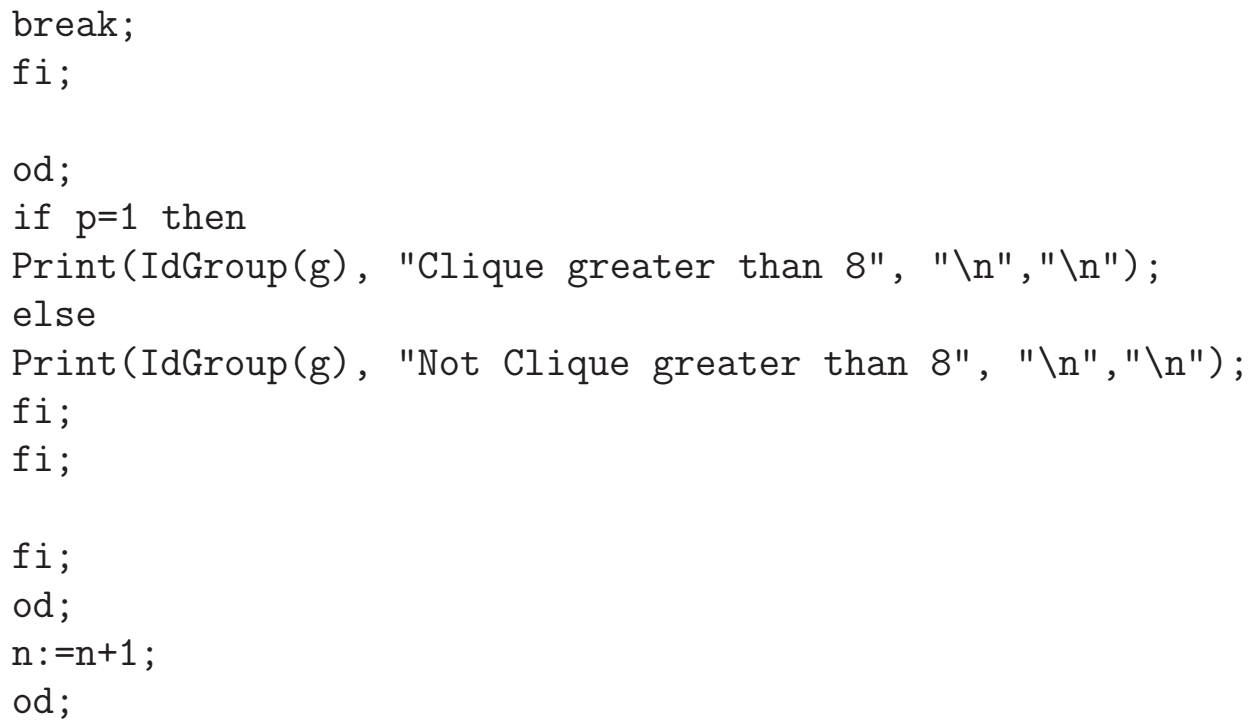

We conclude this section with the following conjecture.

Conjecture 6.5. Let $G$ be a non-solvable group such that $\omega\left(\mathcal{N S}_{G}\right)=8$. Then $G / \operatorname{Sol}(G) \cong A_{5}$.

\section{Groups with the same Non-solvable Graphs}

In [1, Abdollahi et al. conjectured that if $G$ and $H$ are two non-abelian finite groups such that their non-commuting graphs are isomorphic then $|G|=|H|$. They also verified this conjecture for several classes of finite groups. Recently, Nongsiang and Saikia [13] posed similar conjecture for non-nilpotent graphs of finite groups. In this section we consider the following problem.

Problem 7.1. Let $G$ and $H$ be two non-solvable groups such that $\mathcal{N S}_{G} \cong \mathcal{N} \mathcal{S}_{H}$. Determine whether $|G|=|H|$.

We begin the section with the following theorem.

Theorem 7.2. Let $G$ and $H$ be two non-solvable groups such that $\mathcal{N} \mathcal{S}_{G} \cong \mathcal{N} \mathcal{S}_{H}$. If $G$ is finite then $H$ is also finite. Moreover, $|\operatorname{Sol}(H)|$ divides

$$
\operatorname{gcd}\left(|G|-|\operatorname{Sol}(G)|,|G|-\left|\operatorname{Sol}_{G}(x)\right|,\left|\operatorname{Sol}_{G}(g)\right|-|\operatorname{Sol}(G)|\right),
$$

where $g \in G \backslash \operatorname{Sol}(G)$, and hence $|H|$ is bounded by a function of $G$.

Proof. Since $\mathcal{N S}_{G} \cong \mathcal{N} \mathcal{S}_{H}$, we have $|H \backslash \operatorname{Sol}(H)|=|G \backslash \operatorname{Sol}(G)|$ and so $|H \backslash \operatorname{Sol}(H)|$ is finite. If $h \in H \backslash \operatorname{Sol}(H)$ then $\left\{a h a^{-1}: a \in H\right\} \subset H \backslash \operatorname{Sol}(H)$, since $\operatorname{Sol}(H)$ is closed under conjugation. Thus every element in $H \backslash \operatorname{Sol}(H)$ has finitely many conjugates in $H$. It follows that $K=C_{H}(H \backslash \operatorname{Sol}(H))$ has finite index in $H$. Since $\mathcal{N S}_{H}$ has no isolated vertex, there exist two adjacent vertices $u$ and $v$ in $\mathcal{N} \mathcal{S}_{H}$. Now, if $s \in K$ then 
$s \in C_{H}(\{u, v\})$ and so $\langle s u, v\rangle$ is not solvable since $\langle s u, v\rangle \cong\langle u, v\rangle \times\langle s\rangle$. Therefore $K u \subset H \backslash \operatorname{Sol}(H)$ and so $K$ is finite. Hence, $H$ is finite.

It follows that $\operatorname{Sol}(H)$ is a subgroup of $H$ and so $|\operatorname{Sol}(H)|$ divides $|H|-|\operatorname{Sol}(H)|$. Since $|H|-|\operatorname{Sol}(H)|=|G|-|\operatorname{Sol}(G)|$, we have $|\operatorname{Sol}(H)|$ divides $|G|-|\operatorname{Sol}(G)|$. Let $x^{\prime} \in H \backslash \operatorname{Sol}(H)$ and $y \in \operatorname{Sol}_{H}\left(x^{\prime}\right)$. Then, by Lemma 2.6, $\left\langle x^{\prime}, y z\right\rangle$ is solvable for all $z \in \operatorname{Sol}(H)$. Thus $\operatorname{Sol}_{H}\left(x^{\prime}\right)=\operatorname{Sol}(H) \cup y_{1} \operatorname{Sol}(H) \cup \cdots \cup y_{n} \operatorname{Sol}(H)$, for some $y_{i} \in H$. Therefore $|\operatorname{Sol}(H)|$ divides $\left|\operatorname{Sol}_{H}\left(x^{\prime}\right)\right|$ and so $|\operatorname{Sol}(H)|$ divides $|H|-\left|\operatorname{Sol}_{H}\left(x^{\prime}\right)\right|$. We have $\operatorname{deg}\left(\mathcal{N S}_{G}\right)=\operatorname{deg}\left(\mathcal{N S}_{H}\right)$ since $\mathcal{N S}_{G} \cong \mathcal{N} \mathcal{S}_{H}$. Also $\operatorname{deg}(g)=|G|-\left|\operatorname{Sol}_{G}(g)\right|$ for any $g \in V\left(\mathcal{N S}_{G}\right)$ and $\operatorname{deg}(h)=|H|-\left|\operatorname{Sol}_{H}(h)\right|$ for any $h \in V\left(\mathcal{N} \mathcal{S}_{H}\right)$. Therefore $|\operatorname{Sol}(H)|$ divides $|H|-\left|\operatorname{Sol}_{H}(h)\right|$ and hence $|G|-\left|\operatorname{Sol}_{G}(g)\right|$ for any $g \in G \backslash \operatorname{Sol}(G)$. Since $|\operatorname{Sol}(H)|$ divides $|G|-|\operatorname{Sol}(G)|$ and $|G|-\left|\operatorname{Sol}_{G}(g)\right|$, it divides $|G|-|\operatorname{Sol}(G)|-$ $\left(|G|-\left|\operatorname{Sol}_{G}(g)\right|\right)=\left|\operatorname{Sol}_{G}(g)\right|-|\operatorname{Sol}(G)|$. This completes the proof.

Proposition 7.3. Let $G$ be a non-solvable group such that $\mathcal{N S}_{G}$ is finite. Then $G$ is a finite group.

Proof. It follows directly from the first paragraph of the proof of the above theorem.

Proposition 7.4. Let $G$ be a group such that $\mathcal{N S}_{G} \cong \mathcal{N} \mathcal{S}_{A_{5}}$, then $G \cong A_{5}$.

Proof. Since $\mathcal{N S}_{G} \cong \mathcal{N} \mathcal{S}_{A_{5}}$, we have $G$ is a finite non-solvable group and

$$
|G \backslash \operatorname{Sol}(G)|=\left|A_{5} \backslash \operatorname{Sol}\left(A_{5}\right)\right|=59 .
$$

Therefore, $|G|=|\operatorname{Sol}(G)|+59$. Since $\operatorname{Sol}(G)$ is a subgroup of $G$, we have $|\operatorname{Sol}(G)| \leq$ $\frac{|G|}{2}$ and so $|G| \leq 118$. Hence, the result follows.

Remark 7.5. Using the following program in GAP [18], one can see that the nonsolvable graphs of $S L(2,5)$ and $\mathbb{Z}_{2} \times A_{5}$ are isomorphic. It follows that non-solvable graphs of two groups are isomorphic need not implies that their corresponding groups are isomorphic.

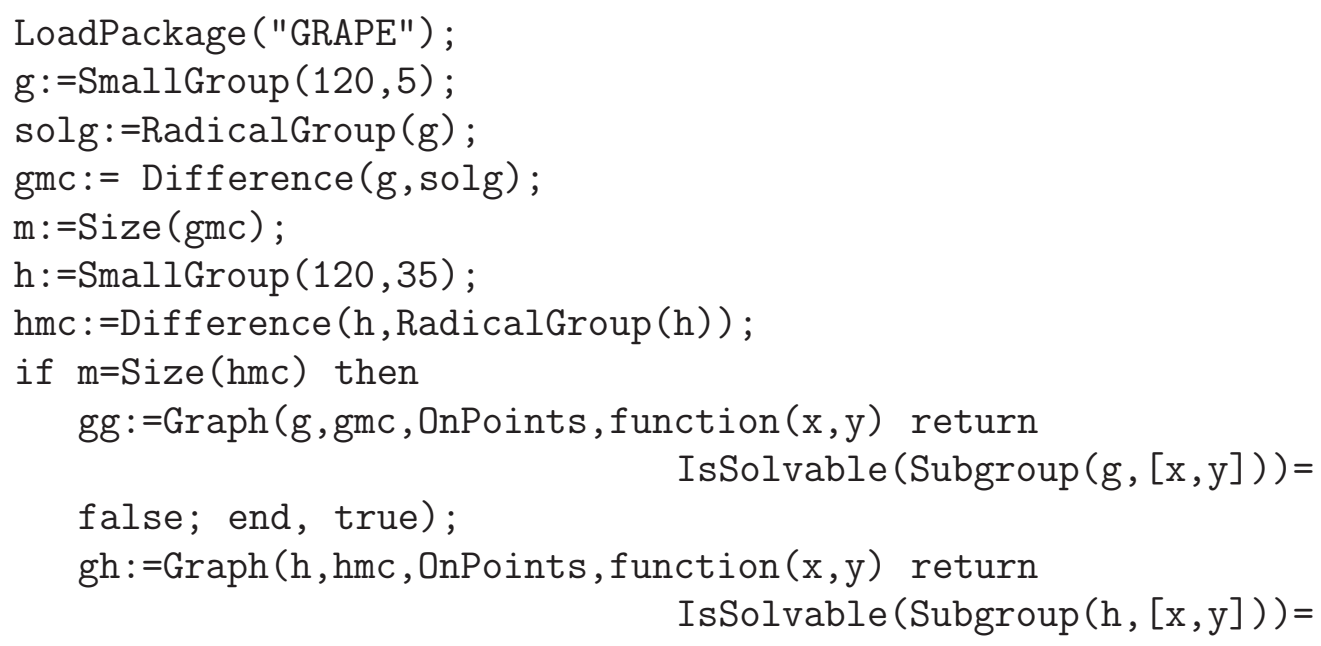




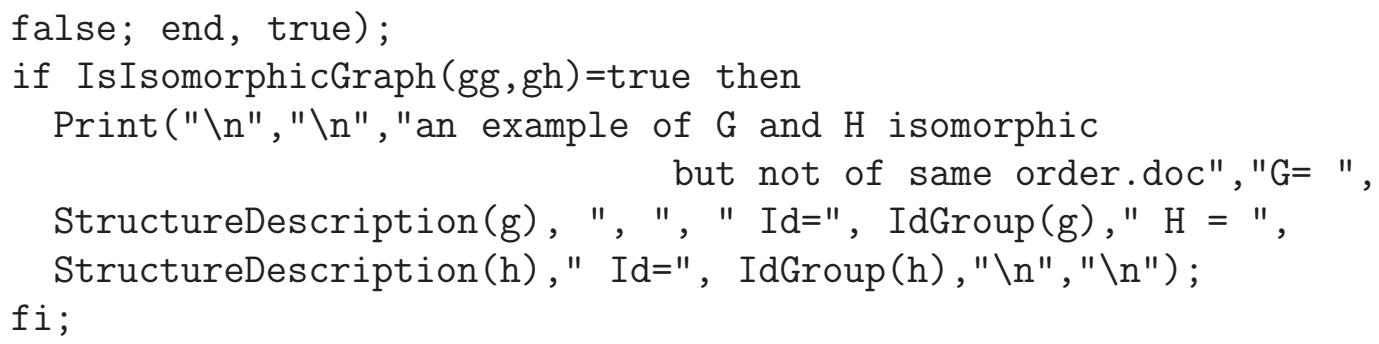

Proposition 7.6. Let $G$ and $H$ be two finite non-solvable groups. If $\mathcal{N S}_{G} \cong \mathcal{N} \mathcal{S}_{H}$ then $\mathcal{N S}_{G \times A} \cong \mathcal{N} \mathcal{S}_{H \times B}$, where $A$ and $B$ are two solvable groups having equal order.

Proof. Let $\varphi: \mathcal{N S}_{G} \rightarrow \mathcal{N S}_{H}$ be a graph isomorphism and $\psi: A \rightarrow B$ be a bijective map. Then $(g, a) \mapsto(\varphi(g), \psi(a))$ defines a graph isomorphism between $\mathcal{N S}_{G \times A}$ and $\mathcal{N} \mathcal{S}_{H \times B}$.

A non-solvable group $G$ is called an $F s$-group if for every two elements $x, y \in G \backslash$

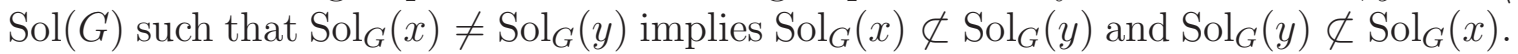

Proposition 7.7. Let $G$ be an Fs-group. If $H$ is a non-solvable group such that $\mathcal{N S}_{G} \cong \mathcal{N} \mathcal{S}_{H}$ then $H$ is also an Fs-group.

Proof. Let $\psi: \mathcal{N S}_{H} \rightarrow \mathcal{N} \mathcal{S}_{G}$ be a graph isomorphism. Let $x, y \in H \backslash \operatorname{Sol}(H)$ such that $\operatorname{Sol}_{H}(x) \subseteq \operatorname{Sol}_{H}(y)$. Then $\psi\left(\operatorname{Sol}_{H}(x) \backslash \operatorname{Sol}(H)\right) \subseteq \psi\left(\operatorname{Sol}_{H}(y) \backslash \operatorname{Sol}(H)\right)$. We have

$$
\psi\left(\operatorname{Sol}_{H}(z) \backslash \operatorname{Sol}(H)\right)=\operatorname{Sol}_{G}(\psi(z)) \backslash \operatorname{Sol}(G) \text { for all } z \in H \backslash \operatorname{Sol}(H) .
$$

Therefore, $\operatorname{Sol}_{G}(\psi(x)) \backslash \operatorname{Sol}(G) \subseteq \operatorname{Sol}_{G}(\psi(y)) \backslash \operatorname{Sol}(G)$. Since $G$ is an Fs-group, we have

$$
\operatorname{Sol}_{G}(\psi(x)) \backslash \operatorname{Sol}(G)=\operatorname{Sol}_{G}(\psi(y)) \backslash \operatorname{Sol}(G) .
$$

It follows that $\operatorname{Sol}_{H}(x) \backslash \operatorname{Sol}(H)=\operatorname{Sol}_{H}(y) \backslash \operatorname{Sol}(H)$ and so $\operatorname{Sol}_{H}(x)=\operatorname{Sol}_{H}(y)$. Hence, $H$ is an Fs-group.

\section{Genus of NON-SOlVABle GRAPH}

The genus of a graph $\Gamma$, denoted by $\gamma(\Gamma)$, is the smallest non-negative integer $g$ such that the graph can be embedded on the surface obtained by attaching $g$ handles to a sphere. Clearly, if $\tilde{\Gamma}$ is a subgraph of $\Gamma$ then $\gamma(\tilde{\Gamma}) \leq \gamma(\Gamma)$. Graphs having genus zero are called planar graphs, while those having genus one are called toroidal graphs. Graphs having genus two are called double-toroidal graphs and those having genus three are called triple-toroidal graph. In [12, Corollary 3.14], it was shown that $\mathcal{N S}_{G}$ is not planar for finite non-solvable group $G$. In this section, we extent [12, Corollary 3.14] and show that $\mathcal{N} \mathcal{S}_{G}$ is neither planar, toroidal, double-toroidal nor triple-toroidal. 
It is well-known (see [16, Theorem 6-39]) that $\gamma\left(K_{n}\right)=\left[\frac{(n-3)(n-4)}{12}\right]$, where $n \geq 3$ and $K_{n}$ is the complete graph on $n$ vertices. Also, if $m, n \geq 2$ then

$$
\gamma\left(K_{m, n}\right)=\left\lceil\frac{(m-2)(n-2)}{4}\right\rceil \text { and } \gamma\left(K_{m, m, m}\right)=\frac{(m-2)(m-1)}{2},
$$

where $K_{m, n}, K_{m, m, m}$ are complete bipartite and tripartite graphs respectively.

Proposition 8.1. Let $G$ be a finite non-solvable group. Then

$$
|\operatorname{Sol}(G)| \leq \sqrt{2 \gamma\left(\mathcal{N S} \mathcal{S}_{G}\right)}+2
$$

Proof. Assume that $Z=\operatorname{Sol}(G)$. By Proposition 6.4, we have $\omega\left(\mathcal{N} \mathcal{S}_{G}\right) \geq 3$. So, there exist $u, v, w \in G \backslash Z$ such that they are adjacent to each other. Then, by Lemma 2.6, $\mathcal{N S}_{G}[u Z \cup v Z \cup w Z]$ is isomorphic to $K_{|Z|,|Z|,|Z|}$. We have

$$
\gamma\left(\mathcal{N S}_{G}\right) \geq \gamma\left(K_{|Z|,|Z|,|Z|}\right)=\frac{(|Z|-2)(|Z|-1)}{2} \geq \frac{(|Z|-2)(|Z|-2)}{2}
$$

and hence the result follows.

Theorem 8.2. Let $G$ be a finite non-solvable graph. Then $\gamma\left(\mathcal{N} \mathcal{S}_{G}\right) \geq 4$. In particular, $\mathcal{N S}_{G}$ is neither planar, toroidal, double-toroidal nor triple-toroidal.

Proof. By Lemma 6.3, we have an element $x \in G \backslash \operatorname{Sol}(G)$ such that $o(x)$ is a prime greater or equal to 5 . Clearly, $\operatorname{nbd}(x) \neq \emptyset$. Assume that $o(y)=2$ for all $y \in \operatorname{nbd}(x)$. Then $x y \in \operatorname{nbd}(x)$ and so $o(x y)=2$. Thus $\langle x, y\rangle=\langle y, x y\rangle$ is isomorphic to a dihedral group, which is a contradiction. Therefore, there exist $y \in \operatorname{nbd}(x)$ such that $o(y) \geq 3$. Let $1 \neq j \in \mathbb{N}$ and $\operatorname{gcd}(j, o(x))=1$. Consider the subsets $H=\left\{x, x^{2}, x^{3}, x^{4}\right\}$, $K=\left\{y^{i} x^{j}: i=1,2, j=0,1,2,3,4\right\}$ of $G \backslash \operatorname{Sol}(G)$ and the induced graph $\mathcal{N S}_{G}[H \cup K]$. Notice that $\mathcal{N S}_{G}[H \cup K]$ has a subgraph isomorphic to $K_{4,10}$ and hence

$$
\gamma\left(\mathcal{N S}_{G}\right) \geq \gamma\left(\mathcal{N S}_{G}[H \cup K]\right) \geq \gamma\left(K_{4,10}\right)=4
$$

This completes the proof.

Remark 8.3. By GAP [18], using the following program, we see that $\mathcal{N S}_{A_{5}}$ has 1140 edges and 59 vertices. Thus by [16, Corollary 6-14], we have $\gamma\left(\mathcal{N S}_{A_{5}}\right) \geq \frac{1140}{6}-\frac{59}{2}+1=$ 161.5 and so $\gamma\left(\mathcal{N S}_{A_{5}}\right) \geq 162$.

LoadPackage ("GRAPE");

$\mathrm{g}:=$ AlternatingGroup (5);

solg: =RadicalGroup $(\mathrm{g})$;

$\mathrm{h}:=\operatorname{Graph}(\mathrm{g}$, Difference ( $\mathrm{g}$, solg) , OnPoints, function (x,y) return

IsSolvable $(\operatorname{Subgroup}(\mathrm{g},[\mathrm{x}, \mathrm{y}]))=$ false; end, true);

$\mathrm{k}:=\operatorname{Vertices}(\mathrm{h})$;

$i:=0$;

for $\mathrm{x}$ in $\mathrm{k}$ do

$i:=i+\operatorname{VertexDegree}(\mathrm{h}, \mathrm{x})$; 
od;

Print ("Number of Edges=",i/2);

Similarly $\mathcal{N S}_{S_{5}}, \mathcal{N} \mathcal{S}_{S L(2,5)}$ and $\mathbb{Z}_{2} \times A_{5}$ has 4560 edges and 119 vertices. So their genera are at least 732 .

A compact surface $N_{k}$ is a connected sum of $k$ projective planes. The number $k$ is called the crosscap of $N_{k}$. A simple graph which can be embedded in $N_{k}$ but not in $N_{k-1}$, is called a graph of crosscap $k$. The notation $\bar{\gamma}(\Gamma)$ stand for the crosscap of a graph $\Gamma$. It is easy to see that $\bar{\gamma}\left(\Gamma_{0}\right) \leq \bar{\gamma}(\Gamma)$ for all subgraphs $\Gamma_{0}$ of $\Gamma$. Also, a graph $\Gamma$, such that $\bar{\gamma}(\Gamma)=1$ is called a projective graph. It is shown in [10] that $2 K_{5}$ is not projective. Hence, any graph containing a subgraph isomorphic to $2 K_{5}$ is not projective. We conclude this paper with the following result.

Theorem 8.4. Let $G$ be a finite non-solvable group. Then $\mathcal{N} \mathcal{S}_{G}$ is not projective.

Proof. As shown in the proof of Theorem 8.2, there exist $x, y \in G \backslash \operatorname{Sol}(G)$ such that $o(x)$ is a prime greater or equal to $5, o(y) \geq 3$ and they are adjacent. Let $1 \neq j \in \mathbb{N}, \operatorname{gcd}(j, o(y))=1$. Consider the subsets $H=\left\{y, x y, x^{2} y, x^{3} y, x^{4} y\right\}$ and $K=\left\{y^{j}, x y^{j}, x^{2} y^{j}, x^{3} y^{j}, x^{4} y^{j}\right\}$ of $G \backslash \operatorname{Sol}(G)$. Then $H \cap K=\emptyset$ and $\mathcal{N S}_{G}[H] \cong$ $\mathcal{N S}_{G}[K] \cong K_{5}$. It follows that $\mathcal{N S}_{G}$ has a subgraph isomorphic to $2 K_{5}$. Hence, $\mathcal{N} \mathcal{S}_{G}$ is not projective.

\section{REFERENCES}

[1] A. Abdollahi, S. Akbari and H. R. Maimani, Non-commuting graph of a group, J. Algebra, 298 (2006), 468-492.

[2] A. Abdollahi, M. Zarrin, Non-nilpotent graph of a group, Comm. Algebra, 38 (2010), 4390-4403.

[3] M. Afkhami, D. G. M. Farrokhi and K. Khashyarmanesh, Planar, toroidal, and projective commuting and noncommuting graphs, Comm. Algebra, 43 (2015), 2964-2970.

[4] B. Akbari, More on the non-solvable graphs and solvabilizers, preprint available at https://arxiv.org/pdf/1806.01012.pdf.

[5] P. Bhowal, D. Nongsiang and R. K. Nath, A note on solvable graphs of finite groups, preprint, available at https://arxiv.org/pdf/1903.01755.pdf.

[6] J. A. Bondy, J. S. R. Murty, Graph Theory with Applications, North-Holland, 1976.

[7] M. R. Darafsheh, H. Bigdely, A. Bahrami and M. D. Monfared, Some results on non-commuting graph of a finite group, Ital. J. Pure Appl. Math., 268 (2014), 371-387.

[8] P. Dutta, J. Dutta and R. K. Nath, Laplacian spectrum of non-commuting graphs of finite groups, Indian J. Pure Appl. Math., 49 (2018), 205-216.

[9] R. Guralnick, B. Kunyavskii, E. Plotkin and A. Shalev, Thompson-like characterizations of the solvable radical, J. Algebra, 300 (2006), 363-375.

[10] H. H. Glover, J. P. Huneke and C. S. Wang, 103 graphs that are irreducible for the projective plane, J. Combinatorial Series B, 27 (1978), 332-370.

[11] R. Guralnick and J. Wilson, The probability of generating a finite soluble group, Proc. London Math. Soc. 81(3) (2000), 405-427.

[12] D. Hai-Reuven, Non-solvable graph of a finite group and solvabilizers, arXiv:1307.2924v1, 2013.

[13] D. Nongsiang and P. K. Saikia, On the non-nilpotent graphs of a group, Int. Electron. J. Algebra, 22 (2017), 78-96. 
[14] D. Nongsiang and P. K. Saikia, On the non-nilpotent graphs of a group, Int. Electron. J. Algebra, 22 (2017), 78-96.

[15] A. A. Talebi, On the non-commuting graphs of group $D_{2 n}$, Int. J. Algebra, 20 (2008), 957-961.

[16] A. T. White, Graphs, Groups and Surfaces, North-Holland Mathematics Studies, no. 8., American Elsevier Publishing Co., Inc., New York, 1973.

[17] J. S. Wilson, The probability of generating a soluble subgroup of a finite group, J. London Math. Soc. (2) 75 (2007), 431-446.

[18] The GAP Group, GAP - Groups, Algorithms, and Programming, Version 4.6.4, 2013 (\protect \vrule width0pt \protect \href\{http://www.gap-system.org\}\{http://www.gap-system.org\}).

Parthajit Bhowal, Department of Mathematical Science, Tezpur University, Napaam784028, Sonitpur, Assam, India.

E-mail address: bhowal.parthajit8@gmail.com

Rajat Kanti Nath, Department of Mathematical Science, Tezpur University, Napaam784028, Sonitpur, Assam, India.

E-mail address: rajatkantinath@yahoo.com

Deiborlang Nongsiang, Department of Mathematics, Union Christian College, Umiam-793122, Meghalaya, India.

E-mail address: ndeiborlang@yahoo.in 